\title{
Bit Error Rate Performance of Quadrature Modulation Transmission Using Reconfigurable Frequency Selective Surfaces
}

\author{
Stephen Henthorn, Kenneth Lee Ford, Senior Member, IEEE, and Timothy O'Farrell
}

\begin{abstract}
A low-complexity radio transmitter is implemented and evaluated using a novel direct antenna modulation (DAM) technique incorporating a reconfigurable antenna. The antenna uses reconfigurable frequency selective surfaces (FSS) to directly phase modulate a radio frequency carrier wave with a quadrature phase shift keying (QPSK) modulation. A hardware-in-the-loop demonstration of the transmitter in a single in single out (SISO) communications system has been developed. To achieve a BER of $10^{-4}$ the proposed system required only $4 \mathrm{dBm}$ extra transmit power compared with instrument grade transmission.
\end{abstract}

Index Terms-Reconfigurable Antennas, Frequency Selective Surfaces, Quadrature Phase Shift Keying

\section{INTRODUCTION}

C ONVENTIONAL radio modulation techniques involve mixing a baseband signal onto a radio frequency (RF) carrier wave at low power, before filtering and amplifying this signal. A disadvantage of this approach is that the requirement for the power amplifiers (PA) is to be linear over a bandwidth comparable with the symbol rate. This requirement often results in the system needing to reduce the amplification due to the increasing bandwidths associated with modern communications systems in order to avoid non-linear effects [1]. Direct antenna modulation (DAM) is a technique where information is modulated onto an RF carrier at or after the antenna, which allows the RF chain to be simplified to a carrier signal that is amplified and fed to the modulating antenna unit. This allows the PA to be narrowband and operate in its more efficient non-linear region while avoiding distortion. The technique does not require any modification of the receiver since a conventional QPSK signal is transmitted.

There have been several approaches to DAM, one being near-field DAM, where an active element surrounded by passive reflectors having their characteristics altered with switches or varactor diodes produces constellation points in the farfield [2]. It can produce arbitrary constellations at the cost of being a complex technique, and is aimed at secure point-topoint communications. Another DAM technique pulse-width modulates the RF feed of two orthogonally oriented antennas with a switch to generate a complex symbol [3]. This has application in a compact, single RF chain MIMO transmitter, in theory achieving arbitrary constellations.

The authors are with the Department of Electrical and Electronic Engineering, University of Sheffield S1 3JD, UK (e-mail: 1.ford, t.ofarrell@sheffield.ac.uk).

This work is funded by an Industrial CASE award from EPSRC with industrial partners NEC and BT. The data reported in this article are available from the ORDA digital repository (https://doi.org/10.15131/shef.data.4802203)
This letter proposes using frequency selective surfaces (FSS) integrated into an antenna to phase modulate a continuous wave $(\mathrm{CW})$ signal, using varactor diodes to alter the phase of the transmitted signal. FSS act as large area LC filters for incident radio waves [4], and are often used as front-end filters in radomes [5]. Reconfigurable FSS have been demonstrated to change the phase of modulated signals previously by integrating variable capacitors, both as a reflective surface to distribute the reflected power of radar signals [6] and as a transmissive layer on buildings to scramble incoming RF communication for security applications [7]. However, no attempt is made in either case to recover the original baseband signal. Modulation with FSS has been proposed in [8], using PIN diodes to amplitude modulate at high frequencies, but no implementation has been shown. This letter presents the first simulated and experimental results of a directly phase modulating antenna using FSS, and is shown implemented in a Direct Sequence Spread Spectrum (DSSS), QPSK, quasi-real time communication system.

\section{Direct Antenna Modulation Radios}

Reconfigurable antennas, and in particular FSS DAM systems, can be used to reduce the complexity of radio transmitters. Fig. 1a shows a conventional quadrature homodyne transmitter, illustrating phase modulation of the carrier by baseband modulated data. A reconfigurable FSS can be used to replace the phase modulation process as the surface acts as an LC bandpass filter for electromagnetic waves while added variable capacitors allow variation of the centre frequency, which changes the phase of a radio carrier signal passing through it (Fig. 1b). The reconfigurable FSS is controlled by an electrical signal derived from the digital data, which varies the voltage across the capacitors. The control signal operates at the data symbol rate to bias the FSS in order to select individual phases $\left(\phi_{m}\right)$ that modulate the amplified continuous wave (CW) RF carrier radiating from a passive antenna.

The direct modulation of the radiated RF carrier replaces the mixing and amplifier circuitry of a conventional quadrature transmitter with a low cost antenna structure and control electronics, the latter operating at the data symbol rate. Also, the FSS configuration allows the use of a narrowband power amplifier operating in its most efficient non-linear region, thereby reducing energy consumption [9] as well as avoiding baseband signal distortion, which leads to spectral regrowth. 


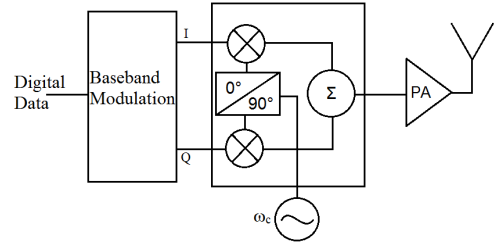

(a)

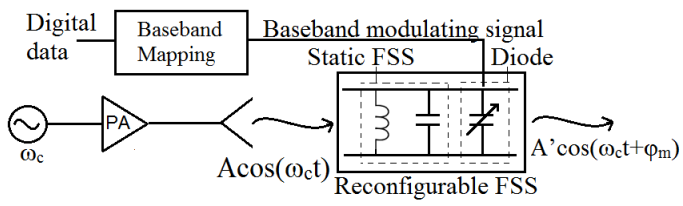

(b)

Fig. 1. (a) Block diagram of a conventional homodyne quadrature transmitter, (b) Concept diagram of a DAM transmitter using a reconfigurable FSS

\section{Reconfigurable Antenna Design}

For the DAM concept to achieve QPSK modulation the FSS is required to provide four orthogonal constellation points where the relative differences in the magnitude of each point is minimised. Secondary requirements for this proof of concept demonstrator are to have minimal insertion loss and broadband performance over the QPSK signal bandwidth. The secondary requirements drive the choice of FSS to be a bandpass filter response which is equivalent to a parallel LC circuit and the FSS unit cell geometry is chosen to be a square loop aperture, Fig. 2a, due to its known wideband properties. For a single layer FSS, the transmission magnitude, at a fixed frequency, will change as the capacitance of the FSS is tuned. However, increasing the number of FSS layers will increase the bandpass filter order of the FSS frequency response which can maintain a more constant magnitude with tuning capacitance. Hence, a trade-off between the number of FSS layers and minimising magnitude variation is required, as illustrated in Fig. 2b which shows the transmission coefficient of an ideal parallel LC FSS which is designed to operate at $1.8 \mathrm{GHz}$ as a function of tuning capacitance. In this simulation up to three FSS layers spaced $\lambda / 4$ apart are shown. The number of layers that might be required for a system implementation will depend on the specifications of that system, however, for this proof of concept three FSS layers was considered adequate giving a potential minimum variation of $1.4 \mathrm{~dB}$ between the QPSK constellation points.

To design the FSS geometry, equations in [10], in combination with Babinet's principle, were used to relate the dimensions of a square-loop geometry to inductance and capacitance values of a parallel resonant circuit. A unit cell was designed to resonate higher than the frequency of interest such that the tuning capacitance of a varactor diode will reduce the resonant frequency. The FSS dimensions in Fig. 2a have an equivalent inductance, $\mathrm{L}$, of $23 \mathrm{pH}$ and capacitance, $\mathrm{C}$, of $85 \mathrm{pF}$ giving a static resonance at $3.6 \mathrm{GHz}$. The three layer FSS design was validated through simulation in CST Microwave Studio where SMV1247 varactor diodes, with equivalent series RLC circuit parameters of $4.9 \Omega, 0.7 \mathrm{nH}$ and tuning capacitance of $0.6 \mathrm{pF}$ -

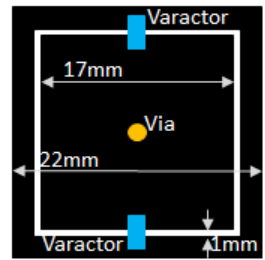

(a)

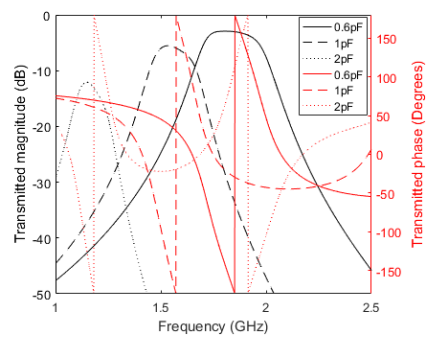

(c)

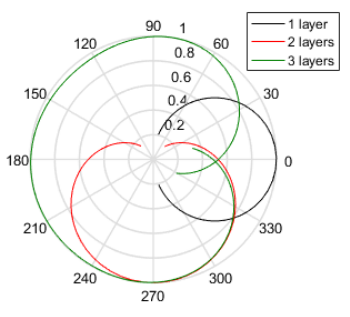

(b)

(d)

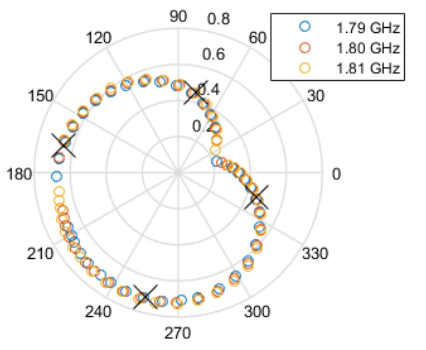

Fig. 2. (a) FSS unit cell, (b) Polar plot with capacitance of transmitted signal of LC bandpass filters of different orders, (c) Simulated transmitted magnitude and phase of FSS, (d) Polar plot with changing capacitance of FSS design over $20 \mathrm{MHz}$ bandwidth

$7 \mathrm{pF}$ were adopted as the tuning element and each FSS layer included a $0.8 \mathrm{~mm}$ thick FR4 substrate. The frequency tunable magnitude and phase of the transmission coefficient as a function of frequency are shown in Fig. 2c, which demonstrate a large phase change in the passband and a minimum insertion loss of $2.9 \mathrm{~dB}$ at $1.8 \mathrm{GHz}$. The complex FSS transmission coefficient can then be mapped to PSK constellation points at the design frequency $(1.8 \mathrm{GHz})$ by varying the diode capacitance. The marked crosses (X) $90^{\circ}$ apart on Fig. 2d, represent an impaired QPSK constellation produced by the FSS which has a maximum $4.2 \mathrm{~dB}$ asymmetry in magnitude. While this may seem large, it was deemed satisfactory for proof-of-concept demonstration as demonstrated by our results.

A reconfigurable antenna incorporating the FSS was then designed. It was found that, to ensure the fields are transmitted through all FSS layers while minimising the antenna size, the FSS should be contained in metal walls. A $4 \times 4$ grid of unit cells, which gives a cavity size slightly larger than $\lambda / 2$, was adopted and a monopole feed was chosen to provide a linearly polarised carrier for demonstration which was placed $\lambda / 4$ from the rear wall to ensure constructive interference of the reflected signal. Fig. 3 shows the final implementation of the antenna. The FSS were fabricated on a substrate with two diodes soldered on each unit cell oriented in the E-plane of the monopole feed, which in Fig. 3b is vertical. On the reverse of each FSS layer is a biasing network of $1 \mathrm{~mm}$ wide horizontal tracks, with vias at the centre of each unit cell to allow them to be biased simultaneously. These add a simulated loss of $0.15 \mathrm{~dB}$ to the system, and raise the FSS resonant frequency by $30 \mathrm{MHz}$. The cavity was fabricated with electrically small apertures to allow electrical connection of the bias signal to the FSS. In comparison to the freespace simulations the intro- 


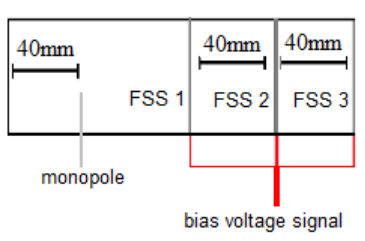

(a)

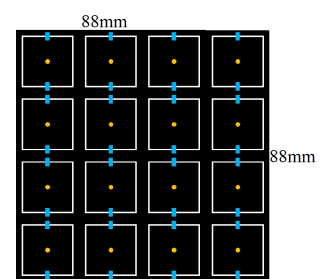

(b)

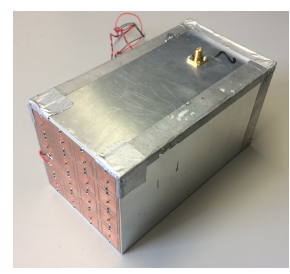

(c)

Fig. 3. Reconfigurable antenna design, (a) Side view, (b) Front view, (c) Photograph

duction of the cavity implementation introduces a frequency shift of $350 \mathrm{MHz}$ which can be mitigated by altering the tuning range of the varactor diodes.

\section{Characterisation OF FSS TRANSMITTER}

The radiation pattern of the antenna was measured in an anechoic chamber at $1.8 \mathrm{GHz}$, with a receiving pyramidal horn antenna $2.5 \mathrm{~m}$ away. The measurements were taken with the FSS biased at four different voltages providing the four points of a QPSK constellation (Fig. 4). The patterns were broadly similar for all bias voltages, but they were all differently attenuated, with a maximum difference of $6.5 \mathrm{~dB}$ at boresight. The measured antenna gain varies from $0.15 \mathrm{dBi}$ to $-6.44 \mathrm{dBi}$, with the low gains arising from a low FSS transmission coefficient and a high varactor diode loss. To avoid a BER degradation at the receiver, a higher average transmit power is required. Simulation of the antenna gives a range of radiation efficiencies across the QPSK constellation points from $-12.8 \mathrm{~dB}$ to $-8.4 \mathrm{~dB}$, which reduces to a range from $-4.3 \mathrm{~dB}$ to $-0.86 \mathrm{~dB}$ with lossless diodes. Therefore, $3.44 \mathrm{~dB}$ of the variation is attributable to the change of the FSS transmission coefficient at each constellation point. Variation of the transmission coefficient can be reduced by having more FSS layers, which increases the FSS bandwidth, but this would reduce the maximum value of the overall transmission coefficient. Designing the FSS with less lossy substrates and tunable components would compensate for the latter.

The switching rate of the FSS was investigated using simulation in CST Design Studio. A square wave operating at a given frequency was inserted across the gap of a unit cell in the corner of a single surface, and the voltages across all diodes recorded. The diodes were modelled as lumped elements with the properties of SMV1247 diodes, as before, but with capacitance held at the highest value required by the constellation, $1.2 \mathrm{pF}$. This showed an underdamped oscillation at $93 \mathrm{MHz}$ that limits the rate of operation to symbol rates below this when using square wave inputs. These effects can

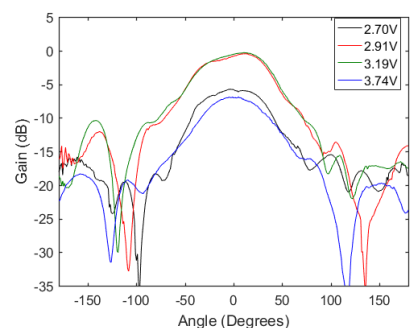

(a)

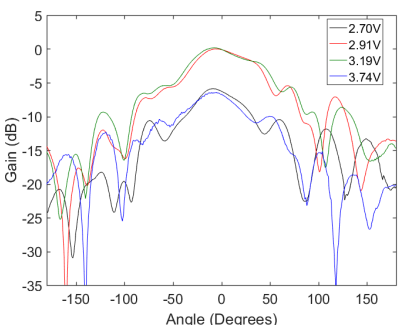

(b)
Fig. 4. Antenna gain pattern in (a) E-plane and (b) H-plane with different biasing voltages

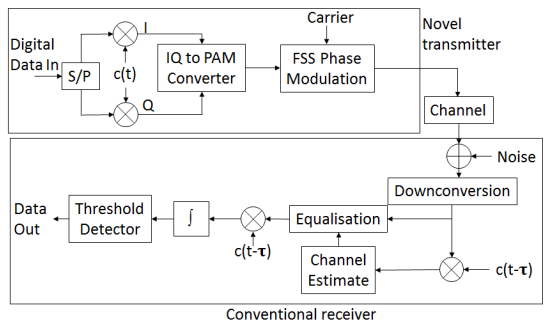

Fig. 5. System schematic of QPSK DSSS communication system with DAM transmitter

be ameliorated by placing a resistor in the feed to the FSS to damp the oscillations and suggests operation in the region of $70 \mathrm{MHz}$ symbol rate is achievable with the current design.

\section{EXPERIMENTAL SYSTEM IMPLEMENTATION}

The FSS transmitter was implemented in an end-to-end SISO communications system (Fig. 5). To ameliorate the FSS' systematic distortion of individual QPSK symbols, DSSS was implemented [11] by multiplying each transmitted I and Q symbol by a balanced bipolar sequence $c(t)$ of length $n$ chips. The spreading results in balanced quantities of systematic but independently distorted chips corresponding to values of a symbol and its complement. Correlation at the receiver (i.e. re-multiplying by $c(t)$ and integrating) averages out the distortion. Owing to its balanced property, a length 15 binary M-sequence was used for $c(t)$. Also, an M-sequence has an impulse-like autocorrelation function, which facilitates channel impulse response (CIR) estimation, whereby the autocorrelation peak identifies the strongest multipath at some chip delay $0 \leq \tau \leq n-1$. The DSSS chips are mapped to a 4-level Pulse Amplitude Modulation (PAM) signal to produce the control signal for the reconfigurable antenna. To estimate the CIR, BPSK pilots, produced by mapping the Msequence to appropriate values in the PAM scheme, prepend the data signal. Data is recovered using a conventional DSSS QPSK receiver and involves estimating the CIR from the pilot symbols, equalising the I and Q symbols, then separately correlating and zero-threshold detecting them.

To evaluate the system bit error rate performance, packets of 222 pilot bits and 888 data bits were used. A Rohde and Schwarz vector signal generator (SMBV1000a) mapped the data packets to digital baseband I and $\mathrm{Q}$ signals. The 


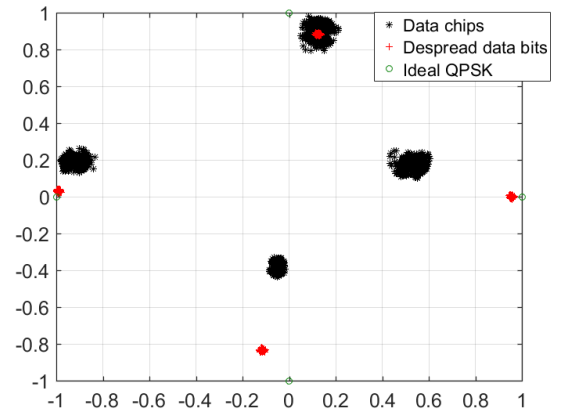

Fig. 6. Normalised constellation diagram of a) Received chips from DAM implementation and b) Data bits after despreading with DSSS

same unit provided a $\mathrm{CW} 1.8 \mathrm{GHz}$ RF carrier signal to the reconfigurable antenna. To convert the digital baseband I and Q signals into a FSS control voltage signal $v(t)$, a comparator on each baseband feed line and a non-linear digital to analogue converter (DAC), consisting of a ladder network of resistors, were implemented. A unity gain buffer was used to isolate the ladder network from the changing impedance of the diodes in the FSS. A passive receiving antenna was placed $0.41 \mathrm{~m}$ away in the far field and was connected to a Rohde and Schwarz FSV30 vector signal analyzer, which was used to sample and acquire the downconverted signal. A PC host running MATLAB was used for post-processing to recover the transmitted data. A nominal symbol rate of $1 \mathrm{MHz}$ was chosen and the measurements were carried out in an echoic laboratory environment.

The equalised chips before correlation and the symbols after correlation are shown as a constellation diagram in Fig. 6, both normalised to their maximum value. There is some phase distortion in the M-sequence chips compared to ideal QPSK constellation points. This is mainly caused by errors in the DAC ladder network attributed to the tolerance of resistor values as well as the large rate of change of phase with respect to changes in bias voltage in some regions of the reconfigurable FSS response. There is also some magnitude variation present, which is inherent in using an FSS as a modulator. However, these distortions are reduced when the balanced spreading sequence is correlated. This is due to each I and Q symbol consisting of an almost equal number of less attenuated and heavily attenuated chips, thereby averaging out the magnitude and phase variation between them.

Fig. 7 plots system bit error rate (BER) versus received signal power for the DAM, DSSS QPSK system. For comparison, the BER performance of instrument grade DSSS QPSK is also shown. The latter is generated by the SMBV100a generator and transmitted through the FSS which is fix biased at 3.3V to achieve maximum transmission. For each power level, 1,127 packets were sent, which amounts to approximately $10^{6}$ data bits being transmitted. The DAM curve follows the expected shape for a line-of-sight AWGN channel, but the FSS transmitter power must be increased by $4 \mathrm{~dB}$ compared with instrument grade modulation to achieve a BER of $10^{-4}$. This is due to the phase error still present on the DSSS plots,

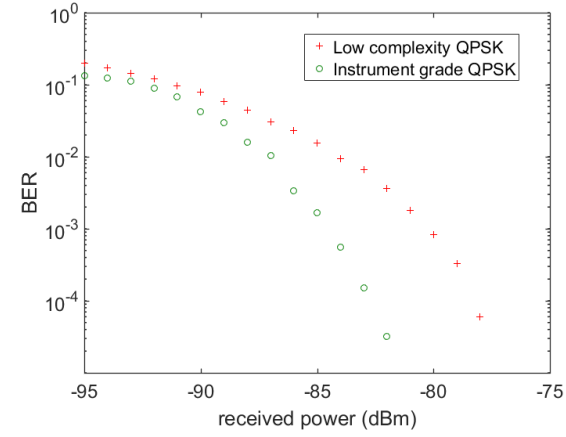

Fig. 7. Bit Error Rate of DAM QPSK (red markers) and instrument grade QPSK (green markers) through reconfigurable antenna at static bias voltage

which could be mitigated by using more accurately calibrated components or by adjusting the decision levels of the threshold detector to ensure the decision is true maximum likelihood.

\section{CONCLUSION}

A low-complexity transmitter based on FSS has been demonstrated in a communication system. The system BER performance of a DSSS QPSK scheme was evaluated and required only a measured $4 \mathrm{~dB}$ increase in transmitted power compared to an instrument grade DSSS QPSK scheme to achieve a BER of $10^{-4}$. Future work will consider the application of the technique to multiple transmitter communication systems, where using multiple reconfigurable antennas with a single RF feed could provide significant implementation cost and space as well as operational energy savings. We will also investigate the power handling capabilities of this technology.

\section{REFERENCES}

[1] A. Raghavan, N. Srirattana, and J. Laskar, Efficiency Enhancement of RF Power Amplifiers. Wiley-IEEE Press, 2008, pp. 173-198.

[2] A. Babakhani, D. B. Rutledge, and A. Hajimiri, "Transmitter architectures based on near-field direct antenna modulation," Solid-State Circuits, IEEE Journal of, vol. 43, no. 12, pp. 2674-2692, 2008.

[3] P. Daehee, K. Minhoe, and C. Dong-Ho, "Novel single-RF mimo system based on repetitive pulse width modulation," Communications Letters, IEEE, vol. 20, no. 1, pp. 165-168, 2016.

[4] B. Munk, Frequency selective surfaces : theory and design. New York: New York : John Wiley, c2000, 2000.

[5] M. Wahid and S. B. Morris, "Band pass radomes for reduced RCS," in Antenna Radar Cross-Section, IEE Colloquium on, Conference Proceedings, pp. 4/1-4/9.

[6] B. Chambers and A. Tennant, "The phase-switched screen," IEEE Antennas and Propagation Magazine, vol. 46, no. 6, pp. 23-37, 2004.

[7] J. Roberts, K. L. Ford, and J. M. Rigelsford, "Secure electromagnetic buildings using slow phase-switching frequency-selective surfaces," $A n-$ tennas and Propagation, IEEE Transactions on, vol. 64, no. 1, pp. 251261, 2016.

[8] G. I. Kiani, T. S. Bird, and K. L. Ford, " $60 \mathrm{GHz}$ ASK modulator using switchable FSS," in Antennas and Propagation Society International Symposium (APSURSI), 2010 IEEE, Conference Proceedings, pp. 1-4.

[9] J. He, P. Loskot, T. O'Farrell, et al "Energy efficient architectures and techniques for Green Radio access networks," in 5th International ICST Conference on Communications and Networking in China (CHINACOM), 2010 IEEE, Conference Proceedings, invited paper.

[10] R. J. Langley and E. A. Parker, "Equivalent circuit model for arrays of square loops," Electronics Letters, vol. 18, no. 7, pp. 294-296, 1982.

[11] T. O'Farrell, "Spread spectrum communication," Jan. 30 2001, US Patent 6,181,729. [Online]. Available: https://www.google.ch/patents/US6181729 